FLASH X-RAY (FXR) ACCELERATOR OPTIMIZATION Electronic Time-Resolved Measurement of $X$-ray Source Size

\author{
J.S. Jacob, M. Ong, P. Wargo
}

November 2, 2004 


\section{Disclaimer}

This document was prepared as an account of work sponsored by an agency of the United States Government. Neither the United States Government nor the University of California nor any of their employees, makes any warranty, express or implied, or assumes any legal liability or responsibility for the accuracy, completeness, or usefulness of any information, apparatus, product, or process disclosed, or represents that its use would not infringe privately owned rights. Reference herein to any specific commercial product, process, or service by trade name, trademark, manufacturer, or otherwise, does not necessarily constitute or imply its endorsement, recommendation, or favoring by the United States Government or the University of California. The views and opinions of authors expressed herein do not necessarily state or reflect those of the United States Government or the University of California, and shall not be used for advertising or product endorsement purposes.

This work was performed under the auspices of the U.S. Department of Energy by University of California, Lawrence Livermore National Laboratory under Contract W-7405-Eng-48. 


\title{
FLASH X-RAY (FXR) ACCELERATOR OPTIMIZATION Electronic Time-Resolved Measurement of X-ray Source Size
}

\author{
J.S. Jacob, M. Ong \\ Lawrence Livermore National Laboratory, 7000 East Ave. L-280 \\ Livermore, CA, 94551 \\ P.Wargo \\ Bechtel Nevada, 161 S. Vasco Rd. Suite A, Livermore, CA 94551
}

\section{Summary}

Lawrence Livermore National Laboratory (LLNL) is currently investigating various approaches to minimize the $\mathrm{x}$ ray source size on the Flash X-Ray (FXR) linear induction accelerator in order to improve $\mathrm{x}$-ray flux and increase resolution for hydrodynamic radiography experiments. In order to effectively gauge improvements to final x-ray source size, a fast, robust, and accurate system for measuring the spot size is required. Timely feedback on x-ray source size allows new and improved accelerator tunes to be deployed and optimized within the limited run-time constraints of a production facility with a busy experimental schedule; in addition, time-resolved measurement capability allows the investigation of not only the time-averaged source size, but also the evolution of the source size, centroid position, and $\mathrm{x}$ ray dose throughout the 70 ns beam pulse. Combined with time-resolved measurements of electron beam parameters such as emittance, energy, and current, key limiting factors can be identified, modeled, and optimized for the best possible spot size.

Roll-bar techniques are a widely used method for x-ray source size measurement, and have been the method of choice at FXR for many years. A thick bar of tungsten or other dense metal with a sharp edge is inserted into the path of the x-ray beam so as to heavily attenuate the lower half of the beam, resulting in a half-light, half-dark image as seen downstream of the roll-bar; by measuring the width of the transition from light to dark across the edge of the roll-bar, the source size can be deduced. For many years, film has been the imaging medium of choice for roll-bar measurements thanks to its high resolution, linear response, and excellent contrast ratio. Film measurements, however, are fairly cumbersome and require considerable setup and analysis time; moreover, with the continuing trend towards all-electronic measurement systems, film is becoming increasingly difficult and expensive to procure.

Here, we shall discuss an x-ray source size measurement system which utilizes a traditional roll-bar setup combined with a high resolution gated CCD camera, fast-response organic plastic scintillator, and image processing and analysis software, which is executable on a standard PC running
LabVIEW and Matlab. Analysis time is reduced from several hours to several minutes, while our experimental results demonstrate good agreement with both traditional film-based roll-bar measurements as well as the entirely unrelated technique of x-ray pinhole camera measurements; in addition, our time-resolved measurements show a significant variation in source size throughout the 70 ns beam pulse, a phenomenon which requires further investigation and indicates the possibility of greatly improving final spot size.

\section{Background on FXR Optimization}

FXR is a linear induction accelerator that produces pulsed x-rays and is used regularly and reliably on explosive experiments since its completion in 1982. In recent years FXR has been incrementally improved, adding double-pulse capability, increasing dose, and reducing x-ray spot-size.

The accelerator generates a $70 \mathrm{~ns}, 3 \mathrm{kA}$ electron beam at $17.5 \mathrm{MeV}$. The $\mathrm{x}$-ray dose at $1 \mathrm{~m}$ is over $400 \mathrm{Rad}$ with a final-focus spot-size of roughly $2.4 \mathrm{~mm}$ FWHM (full-width half-maximum) at the Tantalum bremsstrahlung target [1].

Based on comparison of dose and x-ray spot-size from the Los Alamos National Laboratory (LANL) DARHT and other accelerators, we believe that we could further improve the performance of FXR. In 2001, during the construction of a very large explosive containment facility, the accelerator was not needed for hydrodynamic experiments. We had the opportunity to study the limitations of FXR along with colleagues from the LLNL Beam Research Program.

In general, transverse beam emittance, energy variation, beam motion, and focusing aberrations can all play a role in limiting the electron beam focus [2]. Results from a measurement campaign and computer modeling of the electron beam at the final focus magnet identified energy regulation and beam emittance as the key limiting factors on FXR. Spot size is roughly related to emittance and energy variation by the following equation [3]:

$$
R_{f}^{2} \approx\left(\frac{\varepsilon f}{R_{i}}\right)^{2}+\left(2 \frac{\delta \gamma}{\gamma} R_{i}\right)^{2}
$$


Here, $R_{f}$ is the final spot size, $\varepsilon$ is the emittance, $f$ is the focusing strength of the final focus magnets, $\gamma$ is the relativistic Lorentz factor of the electron beam, and $R_{i}$ is the spot size at the entrance to the final focus magnets. Recently, considerable effort has been invested in time-resolved measurements of both energy variation and emittance. A single-cell test stand has been constructed to investigate cell voltage variations and test improvements to the pulsed power system, as well as to investigate and compensate for beamloading effects in the accelerator cells, which should markedly improve the energy stability of the beam [4,5]. In addition, transverse emittance has been measured using magnet scan [6] and pepper-pot techniques [7] as well as a time-resolved OTR (optical transition radiation) emittance measurement diagnostic [8]. Combined with time-resolved xray spot size data and beam modeling efforts, we should be able to identify key limiting factors and implement an overall plan to improve the $\mathrm{x}$-ray spot size.

\section{Roll-Bar Measurement of X-ray Source Size}

Roll-bar analysis is a widely used technique for measuring x-ray source size, and has been the standard method at FXR for many years. The experimental setup is attractively simple, as shown in Figure 1.

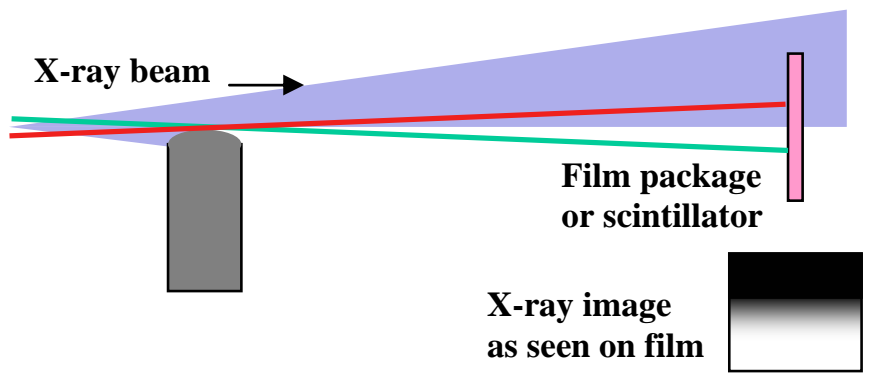

Figure 1. Roll-bar experimental setup

A thick bar of high-Z material such as tungsten or lead is positioned in the bremsstrahlung x-ray path so as to block exactly one-half of the beam in the vertical dimension. The edge of the roll bar is machined to a sharp edge and is positioned with the aid of an alignment laser which is collinear with the axis of the accelerator and, thus, collinear with the $\mathrm{x}$-ray beam. The alignment laser is also used for centering the scintillator or film pack.

X-ray source size analysis is accomplished through taking a vertical cross-section of the x-ray image and measuring the width of the transition from light to dark. An infinitely small point source would produce a step-function transition; with a real beam of finite size, however, the transition occurs over a finite width. Given an x-ray beam with vertical distribution $\mathrm{B}(\mathrm{y})$, as shown in Fig. 2, we can deduce $\mathrm{A}(\mathrm{y})$, the electron distribution at the $\mathrm{x}$-ray source:

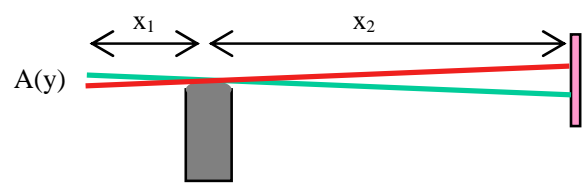

$\mathrm{B}(\mathrm{y})$

Figure 2. X-ray source and image profiles

The magnification of the $\mathrm{x}$-ray source as imaged on the film is given by $M=x_{2} / x_{1}$. For simplicity of analysis, we assume the electron distribution $\mathrm{A}(\mathrm{y})$ is Gaussian; this assumption has been validated by beam profile measurements taken just upstream of the final focus magnets on FXR. Thus, $\mathrm{A}(\mathrm{y})$ takes the form

$$
A(y, \sigma)=\frac{1}{\sqrt{2 \pi} \sigma} \mathrm{e}^{-\frac{1}{2}\left(\frac{\mathrm{y}}{\sigma}\right)^{2}}
$$

$\mathrm{B}(\mathrm{y})$ can be found by integrating across the vertical dimension and accounting for the magnification of the image as viewed at the film:

$$
B(y, \sigma)=\mathrm{M} \int \frac{1}{\sqrt{2 \pi} \sigma} \mathrm{e}^{-\frac{1}{2}\left(\frac{\mathrm{y}}{\sigma}\right)^{2}} d y
$$

Given a measured distribution $\mathrm{B}(\mathrm{y}, \sigma)$, an analytical fit of an error function to the measured data can be performed to determine $\sigma$ and, therefore, the electron beam spot size.

\section{FXR Experimental Setup and Equipment}

Figure 3 shows an overview of the experimental arrangement inside the Contained Firing Facility, while Figure 4 shows an overhead view of the layout. The x-ray conversion target is located immediately behind the "bull nose”.

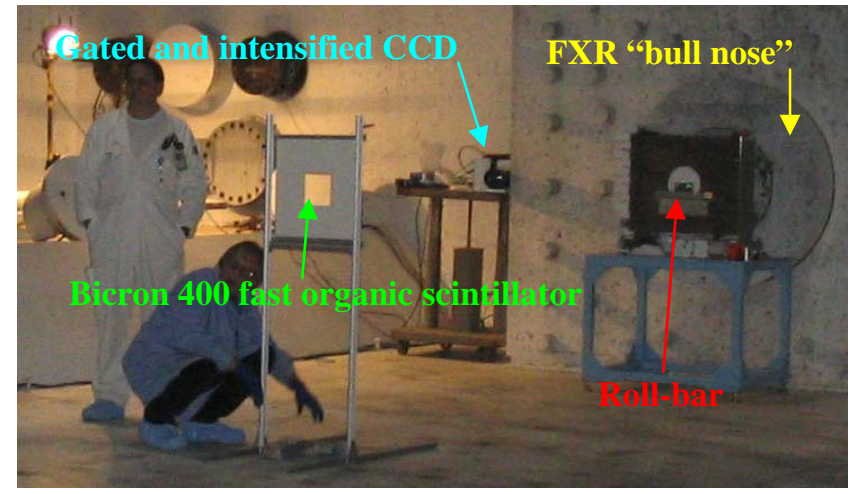

Figure 3. Experimental arrangement inside Contained Firing Facility 


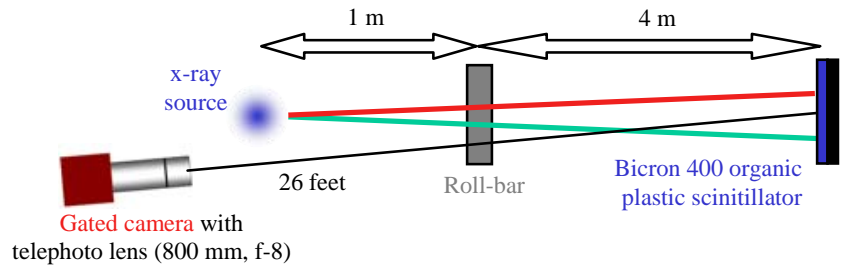

Figure 4. Overhead view of experimental arrangement

An alignment laser is propagated down the axis of the FXR beam pipe and is used to ensure the collinearity of FXR, the roll-bar, and the scintillator, and is also useful for centering the scintillator in the camera's field of view.

Instead of a traditional celluloid film pack, we have made use of a fast-response organic scintillator as our imaging medium:

\begin{tabular}{||l|l|}
\hline Manufacturer \& Model & $\begin{array}{l}\text { Bicron 400 clear organic } \\
\text { plastic }\end{array}$ \\
\hline Rise time & $0.9 \mathrm{~ns}$ \\
\hline Decay time & $2.4 \mathrm{~ns}$ \\
\hline Peak wavelength & $423 \mathrm{~nm}$ (violet) \\
\hline Thickness & $2 \mathrm{~mm}$ \\
\hline Size & $6 \mathrm{x} 6$ inches \\
\hline Light output & $65 \%$ Anthracene \\
\hline
\end{tabular}

Table 1. Scintillator properties [9]

In general, this scintillator was chosen for its fast response time and good light output. Due to the short rise and decay times, we are able to take time-resolved images of the x-ray beam down to 10 ns exposure times. Good light output allows the thickness of the scintillator to be reduced, which increases the image resolution - since scintillation is a volume effect, a thicker scintillator results in more scattering within the material, which blurs the image. The scintillator output spectrum is shown below:

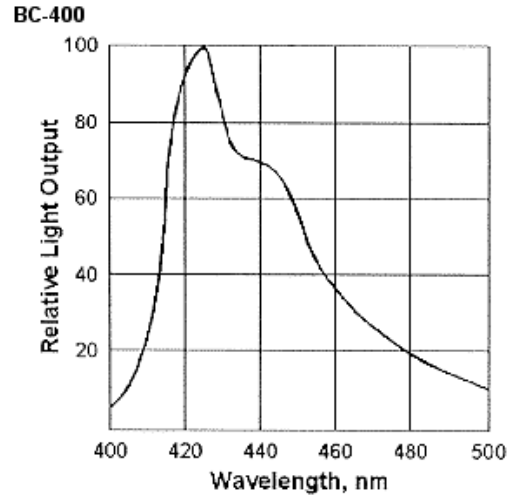

Figure 4. Bicron 400 output spectrum [9]

In order to maximize detection efficiency, the response spectrum of a variety of intensified cameras was compared to the output spectrum of the scintillator. Figure 5 shows the response spectra of several Princeton Instruments cameras:

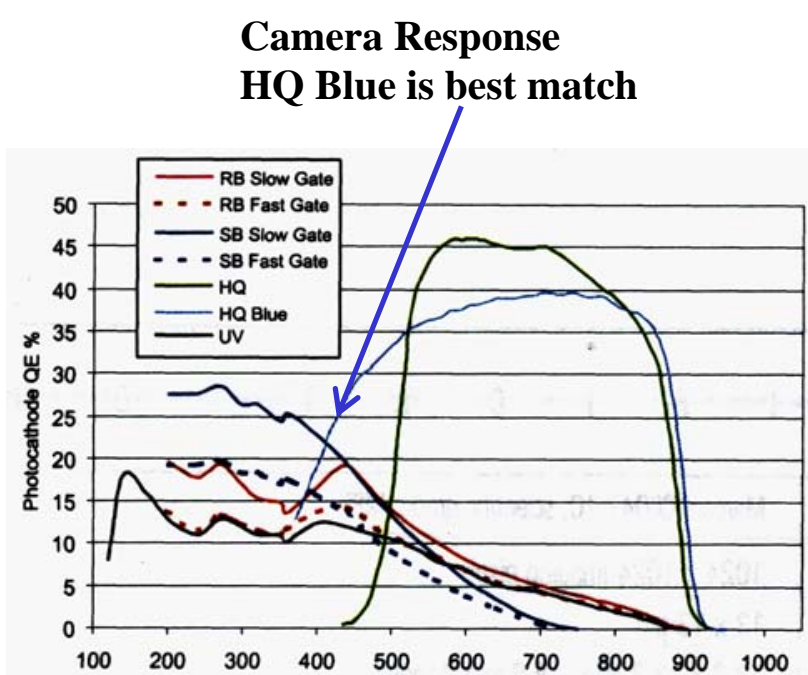

Figure 5. Intensified camera spectral response [10]

The Princeton Instruments PI MAX HQ Blue intensified CCD camera was chosen for its high resolution, fast gating capability, and well-matched spectral response.

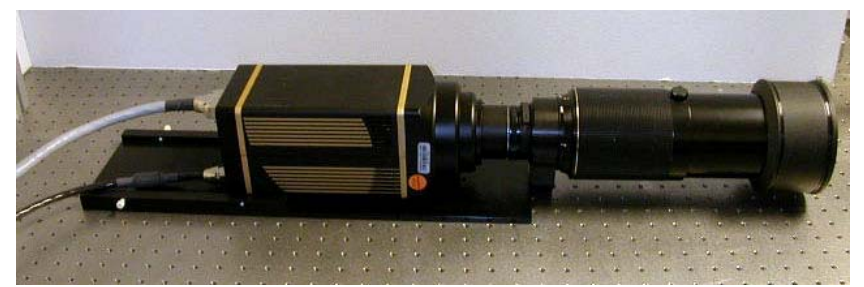

Figure 6. PI MAX HQ Blue intensified CCD camera with lens

\begin{tabular}{||l|l||}
\hline Model & Princeton PI MAX 1KHB \\
\hline Gating & $<5 \mathrm{~ns}$ \\
\hline CCD Cooling & Thermoelectric \\
\hline Resolution & $1024 \times 1024,16$ bits \\
\hline Focal length & $800 \mathrm{~mm}$ \\
\hline Aperture & $\mathrm{f}-8$ \\
\hline
\end{tabular}

Table 2. Camera and lens properties

During actual data collection, the camera is enclosed in a lead "pig" and EMI box for shielding from bremsstrahlung $\mathrm{x}$-rays and electromagnetic interference.

\section{Data Collection and Analysis}

Data collection is accomplished through Roper Scientific's WinView32 software, which provides control over all camera functions and stores the raw image data in the proprietary SPE format, which provides 16 bit dynamic resolution and stores camera parameters along with the image. The first step in data analysis is to capture an image of a known fiducial pattern in order to establish a spatial 
calibration for the optical system. A plastic ruler was affixed to the front of the scintillator and imaged with the camera, as shown below in Figure 7. Measurement of the ruler spacing yielded a spatial calibration of $0.113 \mathrm{~mm} /$ pixel.

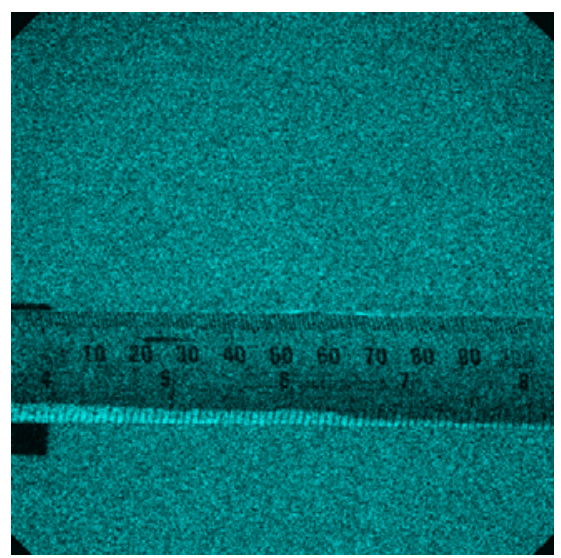

Figure 7. Spatial calibration

Camera triggering is achieved through a series of Stanford Research Systems DG535 digital delay generators, which receive their initial trigger from the FXR master trigger. Due to the limited number of tantalum bremsstrahlung targets available during a given run, initial setup and configuration is accomplished with the beam dump inserted, saving the tantalum targets for actual data collection. Proper timing is verified by taking a 10 ns exposure at the expected center of the beam pulse and verifying the presence of bremsstrahlung x-rays. Next, after any timing issues are resolved, the camera trigger delay is adjusted and an exposure is taken at $40 \mathrm{~ns}$ before and after the expected center of the beam in order to bracket the 70ns beam pulse and verify proper timing. Next, several shots are typically taken to adjust and verify CCD analog gain, MCP timing and gain, detector temperature, and other camera settings. Once the camera setup is verified, the electron beam is delivered to the tantalum target wheel and actual data collection begins.

By adjusting the camera gate width and trigger delay, it is possible to take $10 \mathrm{~ns}$ exposures at various locations in the 70 ns beam pulse, thereby producing a sequence of images that characterize the evolution of the $\mathrm{x}$-ray spot throughout the beam pulse. Average spot size can be calculated by either taking a long exposure that encompasses the entire beam pulse or by calculating the dose-weighted average spot size from 10 ns exposures spanning the entire beam pulse. The dose-weighted average size is given by

$$
\sigma_{\text {Avg. }}=\frac{1}{\text { Dose }_{\text {Total }}} * \sum_{k}\left(\sigma_{k} * \text { Dose }_{k}\right)
$$

Light output of the scintillator is proportional to $\mathrm{x}$-ray dose, and so the relative dose at each point is determined by comparing the average CCD count in a fixed region illuminated by the $\mathrm{x}$-ray beam in each image.
Subsequent to the collection of raw data, the first analysis step is to filter each image to remove unwanted speckle noise caused by scattered x-rays impacting the camera. A specially modified median filter algorithm was developed and implemented in LabVIEW for this purpose. In order to take advantage of the known horizontal symmetry of the data and affect the vertical distribution as little as possible, the filtering algorithm processes a single row at a time. By manual investigation of the raw data, a noise threshold is established. Any pixel with a count above the threshold is considered noise and its value is altered according to a modified median filter -- first, a specified number of the pixel's immediate neighbors to the left and right are examined. Any pixels above the noise threshold are discarded, and the median value of the remaining pixels replaces the value of the original pixel. The image is also rotated to account for a slight tilt of the camera. A block diagram of the filtering algorithm is shown below, as well as a sample data image before and after filtering.

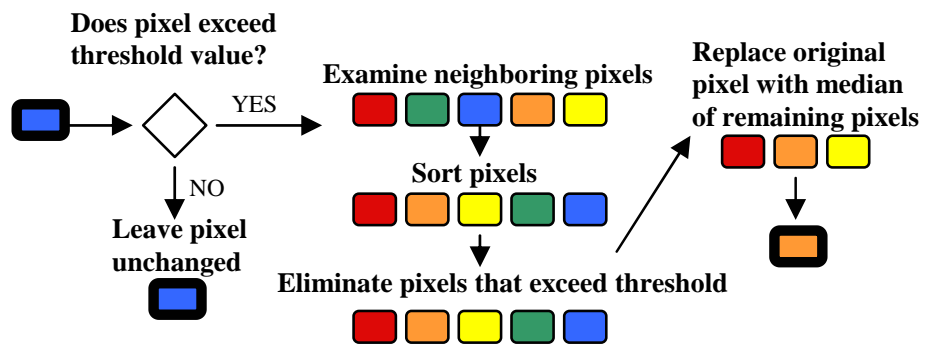

Figure 8. Image filtering algorithm

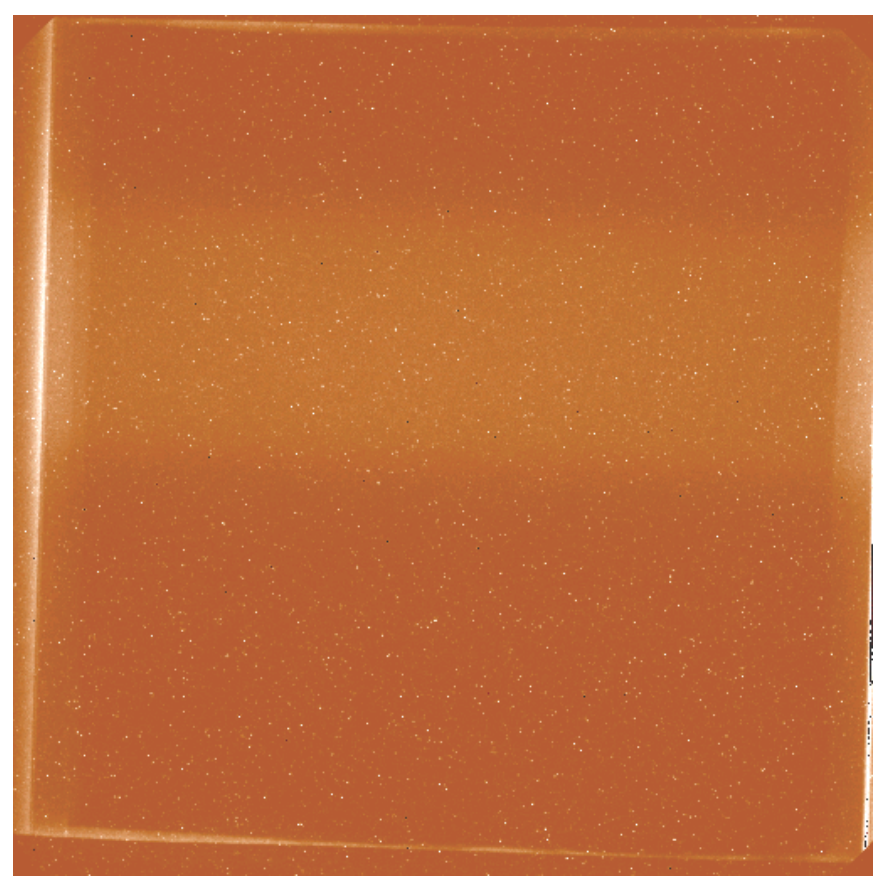

Figure 9. Data image before filtering 


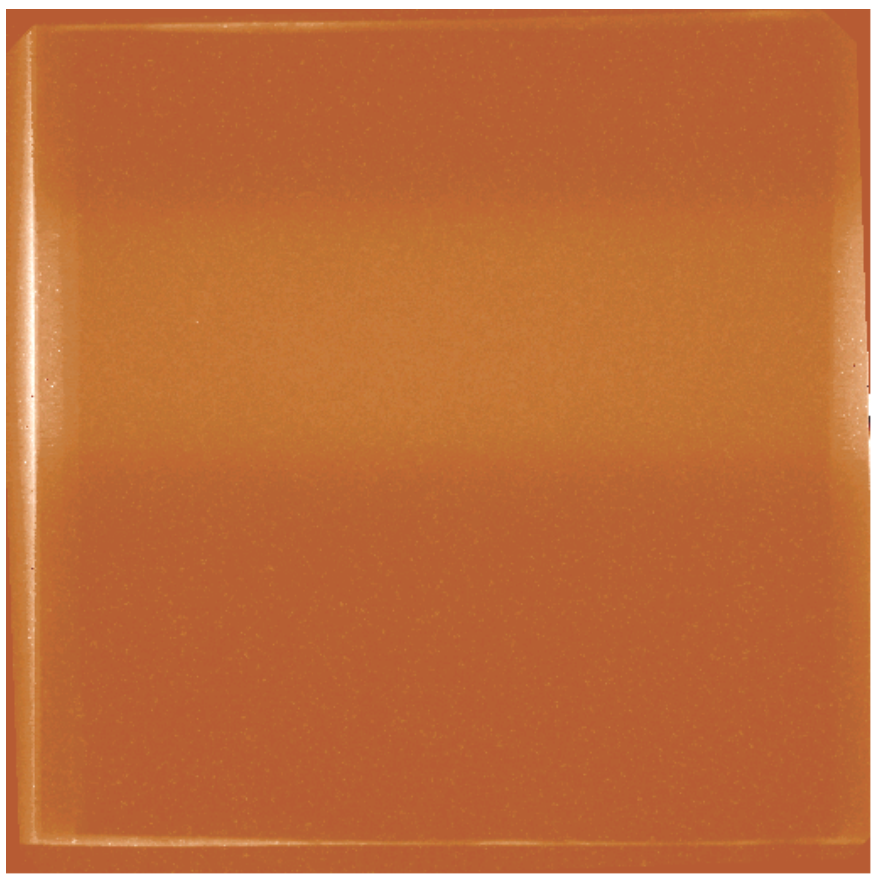

Figure 10. Data image after filtering

After filtering, the images are imported into a Matlab program developed by Roger Richardson and Brian Guidry (LLNL). Given a spatial calibration and a magnification factor, the software takes a vertical cross-section over a specified region of the image and performs a least-squares fit of an error function to the imported data, from which the $\mathrm{x}$ ray source size is calculated. Figure 11 below shows a screen shot of the software, while Figure 12 shows a more detailed view of the data; the blue curve represents the actual empirical data, while the red curve is the best-fit error function.

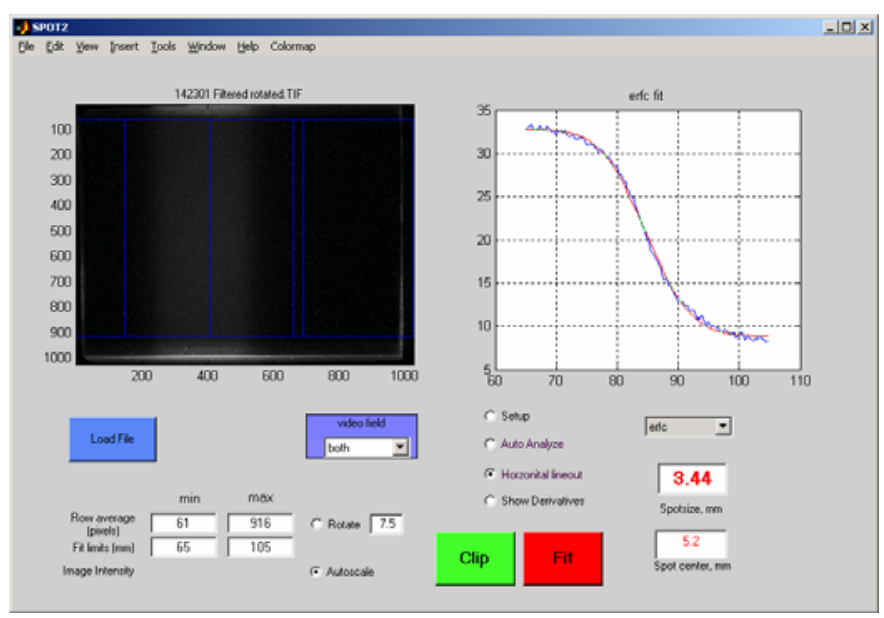

Figure 11. Spot size analysis software

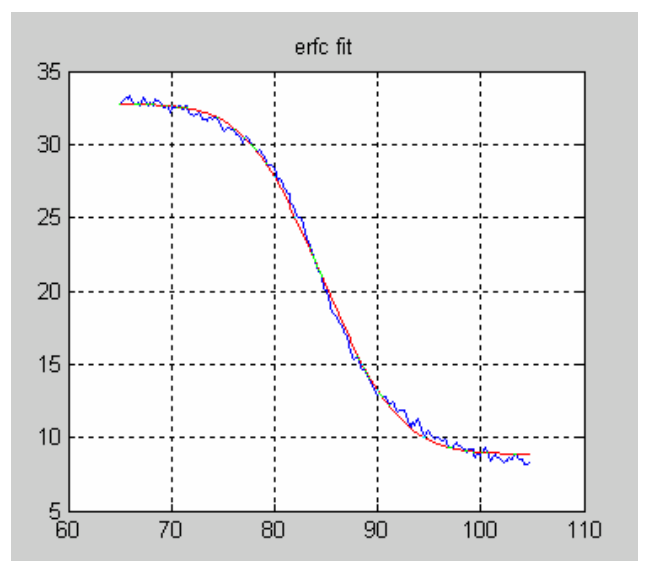

Figure 12. Least-squares error function fit

A series of spot size measurements taken on August 31, 2004, yielded detailed x-ray spot size data for both a normal FXR tune and an experimental tune developed by Steve Falabella (LLNL). The spot size measurement results and actual data images are presented below; note that the relative dose curve corresponds well with the measured beam current, as expected, and confirms a 70 ns FWHM beam pulse. Timeresolved measurements were taken using 10 ns exposures, while entire-beam shots utilized 100 ns exposures.

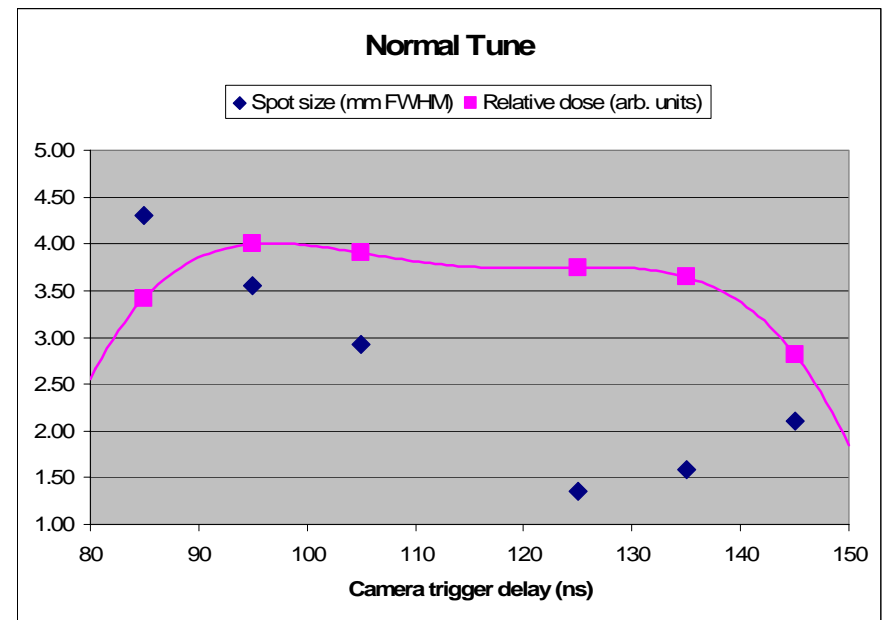

Figure 13. Spot size measurements, 10 ns exposures, normal tune

Clearly, the spot size shows a significant variation throughout the beam pulse. The minimum observed spot size was $1.36 \mathrm{~mm}$ FWHM at a camera trigger delay of $125 \mathrm{~ns}$, and the dose-weighted average spot size was $2.66 \mathrm{~mm}$ FWHM (note that this value is probably artificially high due to the absence of a data point at $115 \mathrm{~ns}$ ). 


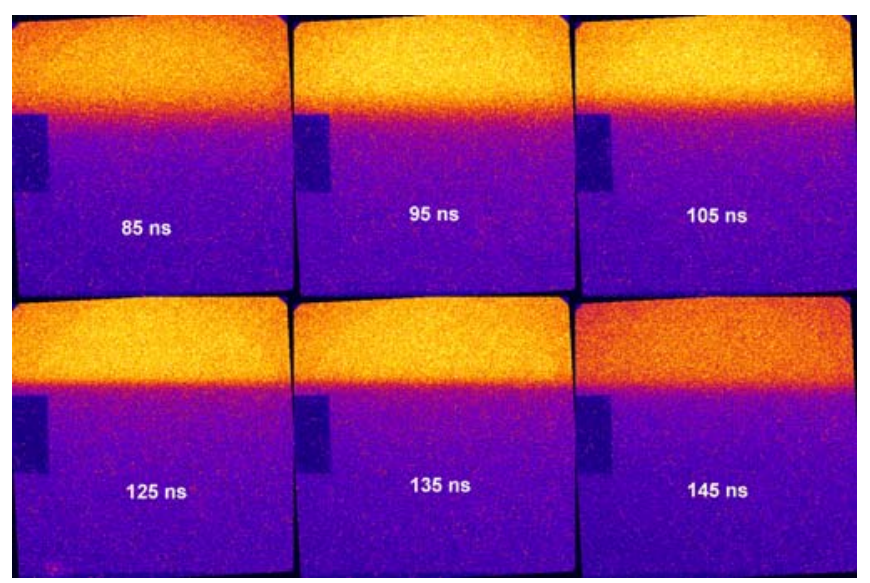

Figure 14. Data images, 10 ns exposures, normal tune

Note that the transition across the roll-bar is clearly visible in the images above and, moreover, it can be qualitatively seen that the width of the transition becomes narrower in the center of the pulse, confirming that the x-ray spot size is indeed smaller at the center of the beam pulse.

Next, a series of long exposure shots was taken to analyze spot size vs. current in FF4, the final focus magnet, with the normal tune. Results and data images are shown below.

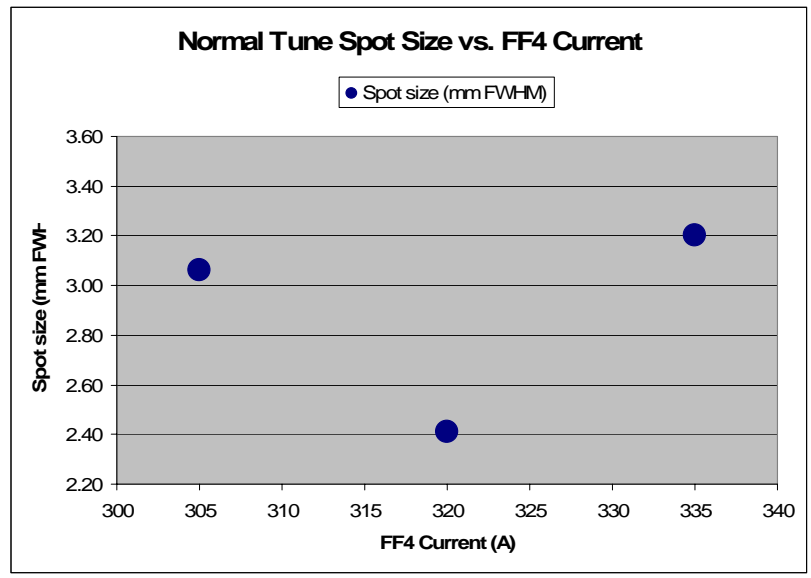

Figure 15. Spot size vs. FF4 current, normal tune, 100 ns exposures

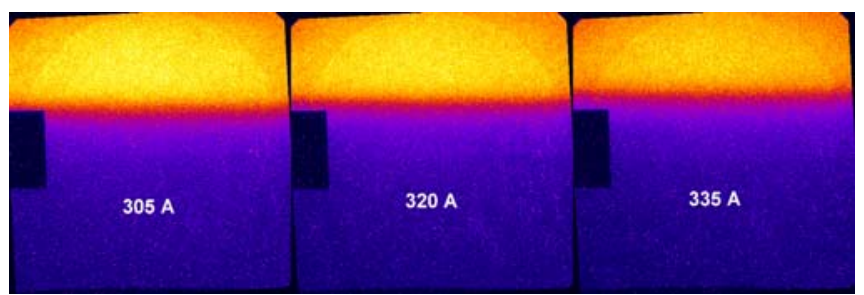

Figure 16. Data images, spot size vs. FF4 current, normal tune, 100 ns exposures

From these data, we can see that the optimal spot size of $2.41 \mathrm{~mm}$ is achieved at FF4 = $320 \mathrm{~A}$, which is the normal current setting.
Next, the accelerator was reconfigured to an experimental tune developed by Steve Falabella. Timeresolved measurements and data images are shown below.

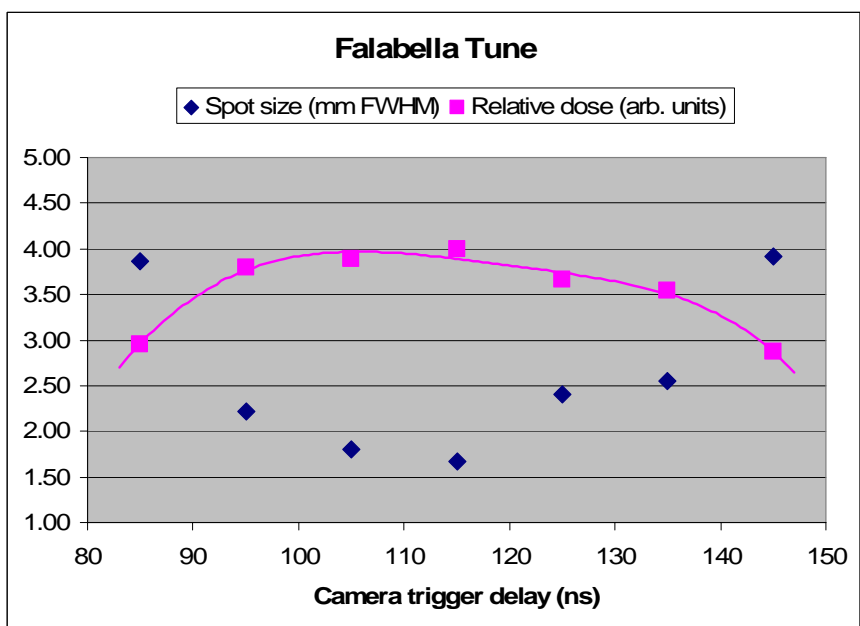

Figure 17. Spot size measurements, 10 ns exposures, Falabella tune

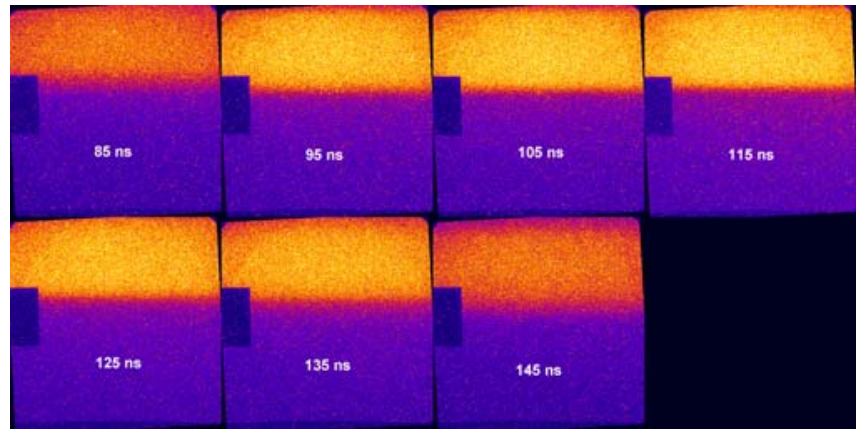

Figure 18. Data images, 10 ns exposures, Falabella tune

Again, we can see that there is a significant variation in spot size throughout the beam pulse; the minimum spot size is not as small as with the normal tune, but the spot appears to remain small for a longer portion of the beam pulse. The minimum observed spot size was $1.67 \mathrm{~mm}$ FWHM at a camera trigger delay of $115 \mathrm{~ns}$, and the dose-weighted average size was $2.53 \mathrm{~mm}$ FWHM.

Finally, another set of long-exposure spot size vs. FF4 measurements was taken using the Falabella tune. Measurements and data images are presented below. 


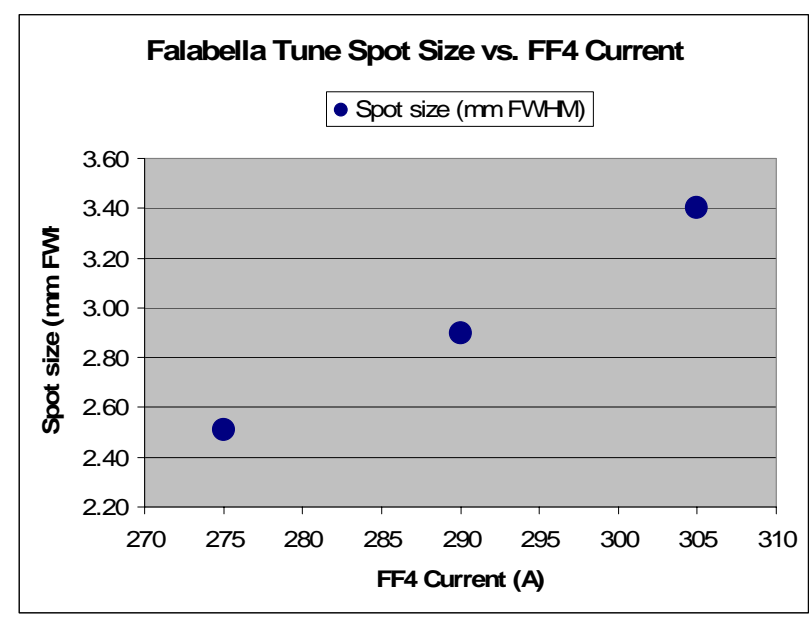

Figure 19. Spot size vs. FF4 current, Falabella tune, 100 ns exposures

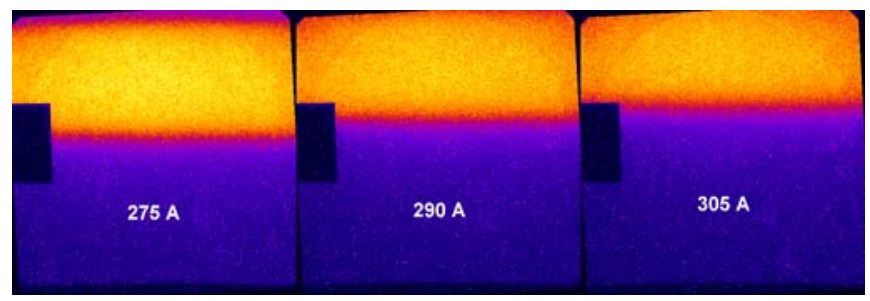

Figure 20. Data images, spot size vs. FF4 current, Falabella tune, 100 ns exposures

From Figure 19, we can see that the best spot size of 2.51 mm FWHM was achieved with FF4 = 275 A; however, from the trend in the data, it is clear that the spot size may be improved by further reducing the FF4 current. Further investigation is warranted.

\begin{tabular}{|c|c|c|}
\hline & Normal tune & Falabella tune \\
\hline $\begin{array}{c}\text { Best long } \\
\text { exposure spot } \\
\text { size }\end{array}$ & $\begin{array}{c}2.41 \mathrm{~mm} \\
\text { FWHM (100 } \\
\text { ns exposure) }\end{array}$ & $\begin{array}{c}2.51 \mathrm{~mm} \\
\text { FWHM (100 } \\
\text { ns exposure) }\end{array}$ \\
\hline $\begin{array}{c}\text { Best short } \\
\text { exposure spot } \\
\text { size }\end{array}$ & $\begin{array}{c}1.36 \mathrm{~mm} \\
\text { FWHM (8 } \\
\text { ns exposure) }\end{array}$ & $\begin{array}{c}1.67 \mathrm{~mm} \\
\text { FWHM (8 ns } \\
\text { exposure) }\end{array}$ \\
\hline $\begin{array}{c}\text { Dose- } \\
\text { weighted } \\
\text { average spot } \\
\text { size }\end{array}$ & $\begin{array}{c}2.66 \mathrm{~mm} \\
\text { FWHM }\end{array}$ & $\begin{array}{c}2.53 \mathrm{~mm} \\
\text { FWHM }\end{array}$ \\
\hline $\begin{array}{l}\text { FF4 setting } \\
\text { for optimal } \\
\text { spot size }\end{array}$ & $320 \mathrm{~A}$ & $275 \mathrm{~A}$ \\
\hline
\end{tabular}

Table 3. Summary of x-ray spot size measurements, August 31, 2004

\section{Acknowledgements}

The authors would like to thank Jan Zentler, Jim Dunlap, Ken Griffin, Keith Lewis, and the entire FXR staff for their continuing help and support. We would also like to thank Roger Richardson and Brian Guidry for their x-ray spot size analysis software.

\section{References}

[1] R. Scarpetti, J. Zentler, J. Boyd, G. Earley, R. Kerr, R. Kihara, K. Griffin, "Upgrades to the LLNL Flash X-Ray Induction Linear Accelerator (FXR)," $11^{\text {th }}$ IEEE International Pulsed Power Conference, Baltimore, MD, June 29-July 2, 1997

[2] G.P. Le Sage, S.G. Anderson, T.E. Cowan, J.K. Crane, T. Ditmire, J.B. Rosenzweig, "RF photoinjector development for a short-pulse, hard X-ray Thomson scattering source," AIP Conference Proceedings, (no 569), p.391-404, 2001

[3] Y. Chen, "Final Focus Spot Size in a Solenoid Focusing System,” UCRL-ID-152620, April 8, 2003

[4] M. Ong, "Flash X-Ray (FXR) Accelerator Optimization Beam-induced Voltage Simulation and TDR Measurements," UCRL-TR-205798, 2004

[5] M. Ong, "Flash X-Ray (FXR) Accelerator Optimization, Injector Voltage-variation Compensation via Beam-induced Gap Voltage,” UCRL-TR-206900, 2004

[6] W.E. Nexsen, R.D. Scarpetti, J. Zentler, "Reconstruction of FXR Beam Conditions," Proceedings of 2001 IEEE Particle Accelerator Conference (2001)

[7] Unpublished measurement by G.A. Westenskow, performed on the FXR injector September 2001

[8] G.P. LeSage, "Time-resolved emittance characterization of an induction linac beam using Optical Transition Radiation,” UCRL-ID-153254, November 5, 2002

[9] Bicron 400 datasheet available online at http://www.detectors.saint-gobain.com

[10] Datasheets for Princeton Instruments cameras available online at

http://www.princetoninstruments.com/products/pimax

[10] P.A. Rodnyi, Physical Processes in Inorganic Scintillators, CRC Press (Boca Raton, 1997)

[11] R.A. Lerche, "Rise time of BC422 plastic scintillator <20PS,” UCRL-JC-106944-ABS-SUM, 1991 